\title{
THE SPECTRAL SEQUENCE OF A COVERING
}

\author{
by D. J. SIMMS \\ (Received 16th December, 1960)
}

1. Introduction.

Let $\mathscr{U}$ be a covering of a topological space $X$ and $\mathscr{F}$ a sheaf of abelian groups over $X$. By a well known result of Leray, (3) II theorems 5.2.4. and 5.4.1., if $\mathscr{U}$ is open, or closed and locally finite, there exists a spectral sequence $\left\{E_{r}\right\}$ satisfying isomorphisms $E_{2}^{p, q} \cong H^{p}\left\{\mathscr{U}, \mathscr{H}^{q}(\mathscr{F})\right\}$ and $E_{\infty}^{p, q} \cong \mathscr{G}^{p} H^{p+q}(X, \mathscr{F})$ for some filtration of the graded group $H^{*}(X, \mathscr{F})$. $\mathscr{H}^{q}(\mathscr{F})$ denotes the system of coefficients over $\mathscr{U}: s \rightarrow H^{q}(|s|, \mathscr{F})$.

In this paper we shall derive another Leray sequence, given in Theorem 1 when $\mathscr{U}$ is locally finite, open or closed, which satisfies isomorphisms $E_{2}^{p, q} \cong$ $H^{p}\left\{\mathscr{U}, \tilde{\mathscr{H}}^{q}(\mathscr{F})\right\}$ and $E_{\infty}^{p, q} \cong \mathscr{G}^{p} \breve{H}^{p+q}(X, \mathscr{F})$ with a suitable filtration of the Cech cohomology $\check{H}^{*}(X, \mathscr{F})$. $\quad \tilde{\mathscr{H}}^{q}(\mathscr{F})$ is the system: $s \rightarrow \tilde{H}^{q}(|s|, \mathscr{F})$, this being the "restricted" cohomology of $|s|$ as a subspace of $X$ introduced in Definition 1 of $\S 2$.

The method used is equivalent to taking the double complex $C^{*, *}\{\mathscr{U}, \mathscr{V} ; \mathscr{F}\}$ defined by a pair of coverings $\mathscr{U}$ and $\mathscr{V}$, (4) p. 220, forming its spectral sequences, and taking their direct limit as $\mathscr{V}$ runs over " all " open coverings of $X$. One of these spectral sequences will degenerate provided $\mathscr{U}$ admits an open refinement; the other will then be the Leray sequence given in Theorem 1 .

In $\S 4$ we express the restricted cohomology $\widetilde{H}^{*}(M, \mathscr{F})$ of a subspace $M \subset X$ as the Cech cohomology of the closure $\bar{M}$ with coefficients in an associated sheaf $\tilde{\mathscr{F}}$ which is the direct image of $\mathscr{F}$ under the inclusion map $M \rightarrow \bar{M}$. In Theorem 2 we obtain a spectral sequence relating the restricted and true cohomologies of $M$, which leads to a sufficient condition for them to be isomorphic.

Finally in Theorem 3 we obtain a map of spectral sequences from the sequence of Theorem 1 to the usual Leray sequence for an open covering, and characterise this map in the $E_{2}$ terms.

\section{Basic Definitions and Operations}

See (3) I 1.6., 2.1., 2.2., 2.6., 3.3., 4.4., 4.5., 4.8., II 5.1., 5.8., (2) V 5., VIII, and (1) XV 5.12.

We denote by $\prod_{I} A_{i}$ the direct product of a family of abelian groups $\left\{A_{i}\right\} i \in I$; by $\lim _{\Lambda} A_{\lambda}$ the direct limit of a direct system of abelian groups $\left\{A_{\lambda}\right\}$ over a 
directed set $\Lambda$; and by $H^{n} A^{*}$ the $n$th cohomology group of a cochain com] $A^{*}=\left(\dot{A}^{n}\right)_{n \in \mathbf{Z}}$.

If $\prod_{I} A_{i}^{*}=\left(\prod_{I} A_{i}^{n}\right)_{n \in Z}$ is the direct product of complexes $A_{i}^{*}$ then $H^{n} \prod_{I} A_{i}$ $\prod_{I} H^{n} A_{i}^{*}$, so that direct products commute with the formation of cohomol groups. If $A_{\lambda}^{*}$ is a direct system of complexes then $H^{n} \lim A_{\lambda}^{*} \cong \lim H^{n} A_{\lambda}{ }^{*}$ that direct limits commute with the formation of cohomology groups.

If $\mathscr{U}=\left\{\mathscr{U}_{i}\right\} i \in I$ is a covering of a topological space $X$ we may call ordered sequence $s=\left(i_{0} i_{1} \ldots i_{p}\right)$ of $(p+1)$ elements of I an ordered $p$-simple: $\mathscr{U}$. We denote by $|s|$ the (possibly empty) set $U_{i_{0}} \cap \ldots \cap U_{i_{p}}$ and by $S_{p}(\mathscr{U})$ set of ordered $p$-simplexes of $\mathscr{U}$. If $\mathscr{F}$ is a sheaf of abelian groups over $X$ any system of local coefficients over $\mathscr{U}$, then the complex $C^{*}(\mathscr{U}, \mathscr{F})$ of coch: of $\mathscr{U}$ with coefficients in $\mathscr{F}$ is defined with $C^{p}(\mathscr{U}, \mathscr{F})=\prod_{s} \mathscr{F}(|s|), s \in S_{p}(\mathscr{U})$.

Let $R(X)$ be the set of all open coverings of $X$ of the form $\mathscr{U}=\left\{U_{x}\right\}$ inde by $x \in X$, such that $x \in U_{x}$ all $x$. Define an ordering relation $\gg$ in $R(X)$ putting $\left\{U_{x}\right\} \gg\left\{V_{x}\right\}$ iff $U_{x} \supset V_{x}$ each $x \in X$. More generally, if $M$ is any sul of $X$, put $\left\{U_{x}\right\} \gg_{M}\left\{V_{x}\right\}$ iff $U_{x} \cap M \supset V_{x} \cap M$ each $x \in X . R(X)$ is a directed with respect to each of the relations $\gg$ and $\gg_{M}$. Let $R_{M}(X)$ be the sel coverings $\left\{V_{x}\right\} \in R(X)$ such that $V_{x} \subset X-M$ if $x \in X-M$. If $M$ is closed it then $R_{M}(X)$ is cofinal in $R(X)$, so that $M \cap R_{M}(X)$ is cofinal in $M \cap R(X)$. $M \cap R_{M}(X)$ with the ordering induced by $\gg_{M}$ may be identified with $R(M)$; thus $R(M)$ is cofinal in $M \cap R(X)$.

$C^{*}(\mathscr{U}, \mathscr{F})$ is a direct system of complexes over $\mathscr{U} \in R(X)$ with the relation and $C^{*}(M \cap \mathscr{U}, \mathscr{F})$ is a direct system over $R(X)$ with each of the relations and $\gg_{M}$, the maps of the system being the same for $\mathscr{U} \gg \mathscr{V}$ as for $\mathscr{U} \gg_{M}$ Moreover $\lim _{\rightarrow} C^{*}(M \cap \mathscr{U}, \mathscr{F}) \cong \lim _{\rightarrow M} C^{*}(M \cap \mathscr{U}, \mathscr{F})$.

Definition 1. If $M$ is any subset of $X$ and $\mathscr{F}$ a sheaf over $M$, then we pr

$$
\tilde{C}^{*}(M, \mathscr{F})=\lim _{\mathscr{U} \in R(X), \triangleright} C^{*}(M \cap \mathscr{U}, \mathscr{F})
$$

and call it the complex of restricted cochains of $M$ (as a subset of $X$ ) with coefficis in $\mathscr{F}$. When $M=X$ we have in particular the Čech complex $\breve{C}^{*}(M, \mathscr{F}$ $\lim _{\mathscr{U} \in R(\mathrm{M})} C^{*}(\mathscr{U}, \mathscr{F})$.

We call the cohomology groups of these complexes the restricted cohomol $\widetilde{H}^{*}(M, \mathscr{F})$ (of $M$ as a subset of $X$ ) and the Cech cohomology $\breve{H}^{*}(M$, respectively. The restricted cohomology is that obtained by using only coch: relative to those coverings of $M$ which can be obtained by intersection $\mathrm{f}_{1}$ coverings of the whole space $X$.

If $\mathscr{U}$ is any covering of $X$ and $\mathscr{F}$ a sheaf over $X$, let $K^{*, *}=C^{*}\left\{\mathscr{U}, \tilde{\mathscr{C}}^{*}\right.$. be the bigraded group defined by:

$$
C^{p}\left\{\mathscr{U}, \tilde{\mathscr{C}}^{q}(\mathscr{F})\right\}=\Pi_{s} \tilde{C}^{q}(|s|, \mathscr{F}), s \in S_{p}(\mathscr{U})
$$


i.e. the group of $p$-cochains of $\mathscr{U}$ with coefficients in the system: $s \rightarrow \widetilde{C}^{q}(|s|, \mathscr{F})$. The differentiations in the complexes $C^{*}\left\{\mathscr{U}, \tilde{\mathscr{C}}^{q}(\mathscr{F})\right\}$ and $C^{p}\left\{\mathscr{U}, \tilde{\mathscr{C}}^{*}(\mathscr{F})\right\}$ define endomorphisms $d^{\prime}$ and $d^{\prime \prime}$ in $K^{*, *}$ of degrees $(1,0)$ and $(0,1)$ with $d^{\prime} d^{\prime \prime}=d^{\prime \prime} d^{\prime}$. If $d_{1}$ and $d_{2}$ are the endomorphisms in $K^{*, *}$ with $d_{1}=d^{\prime}$ and $d_{2}=(-1)^{p} d^{\prime \prime}$ on homogeneous elements of degree $(p, q)$, then $d_{1}^{2}=d_{2}^{2}=d_{1} d_{2}+d_{2} d_{1}=0$, so that $K^{*, *}$ is a double complex with differentiations $d_{1}$ and $d_{2}$.

If $K^{*, *}$ is any double complex and $\left\{{ }^{\prime} E_{r}\right\}$ its spectral sequence with respect to its first filtration, we have isomorphisms ${ }^{\prime} E_{2}^{p, q} \cong{ }^{\prime} H^{p \prime \prime} H^{q} K^{*^{\prime}, *^{\prime \prime}}$ the primes indicating which complexes the cohomology operators act on. If $L^{*} \stackrel{\grave{S}}{\rightarrow} K^{*, *}$ is the inclusion map of the $d_{2} 0$-cocyles of $K^{* * *}$ then:

$$
{ }^{\prime} E_{2}^{n, 0} \cong H^{n \prime \prime} H^{0} K^{*^{\prime}, *^{\prime \prime}} \cong H^{n} L^{*} \text {. }
$$

With this isomorphism the map ' $E_{2}^{n, 0} \rightarrow H^{n}(K)$ defined by the spectral sequence is the same as the induced map of total cohomology:

$$
H^{n} L^{*} \stackrel{j}{\rightarrow} H^{n}\left(K^{*}, *\right)
$$

In particular if the sequence degenerates, i.e. if ' $E_{2}^{p, q} \cong{ }^{\prime} H^{p \prime \prime} H^{q} K^{* \prime}, *^{\prime \prime}=0$ all $q>0$, then (3) will be bijective all $n$. We have a similar result for the inclusion map of the $d_{1} 0$-cocycles.

\section{A Spectral Sequence defined by a Covering}

Lemma 1. If $\left\{M_{i}\right\} i \in I$ is a locally finite family of subsets of a topological space $X$, and if $\left\{\mathscr{V}^{i}\right\} i \in I$ is a family of coverings belonging to $R(X)$; then there exists $\mathscr{U}^{0} \in R(X)$ such that $\mathscr{V}^{i} \gg_{M_{i}} \mathcal{U}^{0}$ each $i \in I$.

Proof. Let $\mathscr{V}^{i}=\left\{V_{x}^{i}\right\}$ each $i$. Let $x \in X$; choose an open neighbourhood $W_{x}$ of $x$ such that $W_{x}$ intersects only a finite number of members of $\left\{M_{i}\right\}$ : $M_{i_{0}}, \ldots, M_{i_{N}}$ say. Put $U_{x}^{0}=W_{x} \cap V_{x}^{i_{0}} \cap \ldots \cap V_{x}^{i_{N}}$. Then $U_{x}^{0} \cap M_{i} \subset W_{x} \cap M_{i}=\varnothing$ if $i \notin\left(i_{0}, \ldots, i_{N}\right)$ and $U_{x}^{0} \subset V_{x}^{i}$ if $i \in\left(i_{0}, \ldots, i_{N}\right)$. Choose $U_{x}^{o}$ similarly for each $x \in X$; then $\mathscr{U}^{0}=\left\{U_{x}^{0}\right\}$ satisfies the required conditions.

Lemma 2. If $\mathscr{U}$ is a locally finite covering of $X$ then for all $p, q \geqq 0$ :

$$
\lim _{\boldsymbol{r}} \prod_{s} C^{p}(|s| \cap \mathscr{V}, \mathscr{F}) \cong \prod_{s} \lim _{\mathscr{r}} C^{p}(|s| \cap \mathscr{V}, \mathscr{F})
$$

over $\mathscr{V} \in R(X)$, $\gg$ and $s \in S_{q}(\mathscr{U})$.

Proof. Let $\theta: \lim _{\gg} \prod_{s} C^{p}(|s| \cap \mathscr{V}, \mathscr{F}) \rightarrow \prod_{s} \lim _{\gg_{|s|}} C^{p}(|s| \cap \mathscr{V}, \mathscr{F})$ be the homomorphism defined by $\theta\left[\lim _{\rightarrow} \prod_{s} c^{p}\left(s, \mathscr{V}^{0}\right)\right]=\prod_{s} \lim _{D_{1}, 1} c^{p}\left(s, \mathscr{V}^{0}\right)$ for any set of elements $c^{p}\left(s, \mathscr{V}^{0}\right) \in C^{p}\left(|s| \cap \mathscr{V}^{0}, \mathscr{F}\right)$. By the limit of an element we mean its projection in the limit group.

Then $\lim _{\rightarrow} \prod_{s} c^{p}\left(s, \mathscr{V}^{0}\right) \in$ kernel $\theta \Rightarrow \lim _{D_{1}, 1} c^{p}\left(s, \mathscr{V}^{0}\right)=0$ all $s \in S_{q}(\mathscr{U}) \Rightarrow$ for all $s, \exists \mathscr{V}^{s} \in R(X)$ such that $\mathscr{V}^{0} \gg_{|s|} \mathscr{\mathscr { V }}^{s}$ and $\pi_{\mathscr{V}^{s}}^{\mathscr{V}^{0}}\left[c^{p}\left(s, \mathscr{V}^{0}\right)\right]=0$, where $\left\{\pi_{\mathscr{V}^{\prime \prime}}^{\mathscr{V}^{\prime \prime}}\right\}$ are the maps of the direct systems involved. But $\mathscr{U}$ is locally finite, so 
that the collection of subsets $\{|s|\}$ for $s \in S_{q}(\mathscr{U})$ is locally finite, and therefore by Lemma 1 there exists $\mathscr{U}^{0} \in R(X)$ with $\mathscr{V}^{s} \gg|s| \mathscr{U}^{0}$ all $s \in S_{q}(\mathscr{U})$; we can also

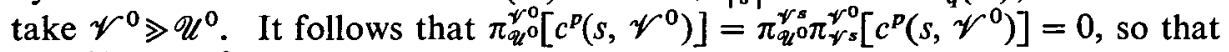
$\lim _{\rightarrow} \prod_{s} c^{p}\left(s, \mathscr{V}^{0}\right)=0$ and $\theta$ is a monomorphism.

Also if $\prod_{s} \lim _{D_{\mid} s \mid} c^{p}\left(s, \mathscr{V}^{s}\right) \in \prod_{s} \lim _{\gg_{1} \mid} C^{p}(|s| \cap \mathscr{V}, \mathscr{F})$ then by Lemma 1 there exists $\mathscr{U}^{0} \in R(X)$ with $\mathscr{V}^{s} \gg_{|s|} \mathscr{U}^{0}$ each $s$. Thus

$$
\begin{aligned}
& \prod_{s} \lim _{>|s|} c^{p}\left(s, \mathscr{V}^{s}\right)=\prod_{s} \lim _{>_{1} s \mid}\left\{\pi_{\mathscr{R}^{\circ}}^{\mathscr{V}^{s}}\left[c^{p}\left(s, \mathscr{V}^{s}\right)\right]\right\} \\
& =\theta\left[\lim \prod_{s}\left\{\pi_{\mathscr{U}^{\prime}}^{\mathscr{Y}^{s}} c^{p}\left(s, \mathscr{V}^{s}\right)\right\}\right]
\end{aligned}
$$

which shows that $\theta$ is also an epimorphism.

The isomorphism

$$
\theta: \lim _{\gg} \prod_{s} C^{p}(|s| \cap \mathscr{V}, \mathscr{F}) \cong \prod_{s} \lim _{>_{|s|}} C^{p}(|s| \cap \mathscr{V}, \mathscr{F})
$$

together with the isomorphisms

prove the lemma.

$$
\lim _{\gg} C^{p}(|s| \cap \mathscr{V}, \mathscr{F}) \cong \lim _{\gg_{|s|}} C^{p}(|s| \cap \mathscr{V}, \mathscr{F})
$$

We are now in a position to obtain a Leray sequence for a locally finite covering, as follows.

Theorem 1. If $\mathscr{U}$ is a locally finite, open or closed, covering of a topological space $X$, admitting an open refinement, and if $\mathscr{F}$ is a sheaf of abelian groups over $X$; then the Čech cohomology group $\breve{H}^{*}(X, \mathscr{F})$ has a filtration so that there exists a spectral sequence $\left\{E_{r}\right\}$ with isomorphisms

and

$$
E_{2}^{p, q} \cong H^{p}\left\{\mathscr{U}, \tilde{\mathscr{H}}^{q}(\mathscr{F})\right\}
$$

$$
E_{\infty}^{p, q} \cong \mathscr{G}^{p} \check{H}^{p+q}(X, \mathscr{F})
$$

all $p, q \geqq 0 ;$ where $\tilde{\mathscr{H}}^{q}(\mathscr{F})$ denotes the system of coefficients : $s \rightarrow \tilde{H}^{q}(|s|, \mathscr{F})$ (see Definition 1 of $\S 2$ ).

Proof. Let $K^{*, *}=C^{*}\left\{\mathscr{U}, \tilde{\mathscr{C}}^{*}(\mathscr{F})\right\}$ be the double complex introduced in (2), and let ' $E(K)$ and " $E(K)$ be its two spectral sequences; see (3) I 4.8. We shall show that " $E(K)$ degenerates and that ' $E(K)$ fulfills the requirements of the theorem.

In the first place

$$
\begin{aligned}
H^{q} C^{p}\left\{\mathscr{U}, \tilde{\mathscr{C}}^{*}(\mathscr{F})\right\} & =H^{q} \prod_{s} \tilde{C}^{*}(|s|, \mathscr{F}) \quad s \in S_{p}(\mathscr{U}) \\
& \cong \prod_{s} \tilde{H}^{q}(|s|, \mathscr{F}),
\end{aligned}
$$

since direct products commute with the formation of cohomology groups; thus

$$
H^{q} C^{p}\left\{\mathscr{U}, \tilde{\mathscr{C}}^{*}(\mathscr{F})\right\} \cong C^{p}\left\{\mathscr{U}, \tilde{\mathscr{H}}^{q}(\mathscr{F})\right\}
$$


and hence

$$
\begin{aligned}
{ }^{\prime} E_{2}^{p, q}(K) & \cong{ }^{\prime} H^{p \prime \prime} H^{q} C^{*^{\prime}}\left\{\mathscr{U}, \tilde{\mathscr{C}}^{* \prime}(\mathscr{F})\right\} \\
& \cong{ }^{\prime} H^{p} C^{* \prime}\left\{\mathscr{U}, \tilde{\mathscr{H}}^{q}(\mathscr{F})\right\} \\
& =H^{p}\left\{\mathscr{U}, \tilde{\mathscr{H}^{q}}(\mathscr{F})\right\} .
\end{aligned}
$$

The primes indicate which complexes the cohomology operators act on. Moreover

$$
\begin{aligned}
C^{q}\left\{\mathscr{U}, \tilde{\mathscr{C}}^{p}(\mathscr{F})\right\} & =\prod_{s} \tilde{C}^{p}(|s|, \mathscr{F}) \quad s \in S_{q}(\mathscr{U}) \\
& =\prod_{s} \lim _{\mathscr{V} \in R(X)} C^{p}(|s| \cap \mathscr{V}, \mathscr{F}) \\
& \cong \lim _{\mathscr{V}} \prod_{s} C^{p}(|s| \cap \mathscr{V}, \mathscr{F})
\end{aligned}
$$

by Lemma 2 , since $\mathscr{U}$ is locally finite; therefore

$$
\begin{aligned}
C^{q}\left\{\mathscr{U}, \tilde{\mathscr{C}}^{p}(\mathscr{F})\right\} & \cong \lim _{\mathscr{V}} \prod_{s} \prod_{t} \mathscr{F}(|s| \cap|t|) \quad t \in S_{p}(\dot{\mathscr{V}}) \\
& \cong \lim _{\mathscr{V}} \prod_{t}\left\{\prod_{s} \mathscr{F}(|s| \cap|t|)\right\} \\
& =\lim _{\boldsymbol{v}} \prod_{t} C^{q}(|t| \cap \mathscr{U}, \mathscr{F}) ;
\end{aligned}
$$

so that

$$
\begin{aligned}
H^{q} C^{*}\left\{\mathscr{U}, \tilde{\mathscr{C}}^{p}(\mathscr{F})\right\} & \cong H^{q} \lim _{\mathscr{t}} \prod_{t} C^{*}(|t| \cap \mathscr{U}, \mathscr{F}) \\
& \cong \lim _{\mathscr{V}} \prod_{t} H^{q}(|t| \cap \mathscr{U}, \mathscr{F}) \\
& =0 \quad(\text { all } q>0),
\end{aligned}
$$

since $\mathscr{U}$ admits an open refinement and hence a refinement by an element $\mathscr{V} \in R(X)$. For such an element $|t| \cap \mathscr{U}$ is a trivial covering of $|t|$ each $t \in S_{p}(\mathscr{V})$ and therefore $H^{q}(|t| \cap \mathscr{U}, \mathscr{F})=0$ for all $q>0$.

Also

Thus

$$
\begin{aligned}
H^{o} C^{*}\left\{\mathscr{U}, \tilde{\mathscr{C}}^{p}(\mathscr{F})\right\} & \cong \lim _{\mathscr{r}} \prod_{t} H^{o}(|t| \cap \mathscr{U}, \mathscr{F}) \\
& \cong \lim _{\mathscr{V}} \prod_{t} \mathscr{F}(|t|) \quad \text { by (3) II 5.2.2. } \\
& =\lim _{\mathscr{r}} C^{p}(\mathscr{V}, \mathscr{F}) \\
& =\check{C}^{p}(X, \mathscr{F}) .
\end{aligned}
$$

$$
\begin{aligned}
{ }^{\prime \prime} E_{2}^{p, q}(K) & \cong{ }^{\prime} H^{p \prime \prime} H^{q} C^{* "}\left\{\mathscr{U}, \tilde{\mathscr{C}}^{*^{\prime}}(\mathscr{F})\right\} \\
& =0 \quad(\text { all } q>0)
\end{aligned}
$$


and

$$
\begin{aligned}
" E_{2}^{n, o}(K) & \cong{ }^{n n} H^{\prime \prime} H^{o} C^{* \prime}\left\{\mathscr{U}, \tilde{\mathscr{C}}^{*^{\prime}}(\mathscr{F})\right\} \\
& \cong H^{n} \check{C}^{*^{\prime}}(X, \mathscr{F}) \\
& =\check{H}^{n}(X, \mathscr{F}) .
\end{aligned}
$$

This shows that the spectral sequence " $E(K)$ degenerates, giving isomorphisms (1) XV 5.12.:

$$
\breve{H}^{*}(X, \mathscr{F}) \cong " E_{2}^{*, 0}(K) \cong H^{*}(K),
$$

this being the total cohomology of the double complex $K^{*, *}$, and giving $\breve{H}^{*}(X, \mathscr{F})$ two filtrations induced by those of $K$.

Finally

$$
\begin{aligned}
{ }^{\prime} E_{\infty}^{p, q}(K) & \cong{ }^{\prime} G^{p} H^{p+q}(K) \quad \text { see (3) I 4.2.2. } \\
& \cong{ }^{\prime} \mathscr{G}^{p} \breve{H}^{p+q}(K),
\end{aligned}
$$

which completes the proof that $E(K)$ is a spectral sequence satisfying the required conditions.

\section{The Restricted Cohomology of a Subspace}

The restricted cochains of a subspace $M \subset X$, with coefficients in a sheaf $\mathscr{F}$ over $M$, can be expressed as Cech cochains of the closure $\bar{M}$ with coefficients in an associated sheaf $\tilde{\mathscr{F}}$ as follows. Let $\tilde{\mathscr{F}}$ be the direct image (3) II 1.13. of $\mathscr{F}$ under the inclusion map $i: M \rightarrow \bar{M}$; this is the sheaf defined by $\tilde{\mathscr{F}}(\bar{M} \cap U)$ $=\mathscr{F}(M \cap U)$ for open sets $U$ of $X$. If $\mathscr{V}$ is an open covering of $X$ then

$$
\begin{aligned}
C^{p}(M \cap \mathscr{V}, \mathscr{F}) & \cong \prod_{s} \mathscr{F}(M \cap|s|) \quad s \in S_{p}(\mathscr{V}) \\
& =\prod_{s} \tilde{\mathscr{F}}(\bar{M} \cap|s|) \\
& \cong C^{p}(\bar{M} \cap \mathscr{V}, \tilde{\mathscr{F}})
\end{aligned}
$$

giving an isomorphism of complexes $C^{*}(M \cap \mathscr{V}, \mathscr{F}) \cong C^{*}(\bar{M} \cap \mathscr{V}, \tilde{\mathscr{F}})$. So that

$$
\begin{aligned}
\tilde{C}^{*}(M, \mathscr{F}) & =\lim _{\mathscr{V} \in R(X)} C^{*}(M \cap \mathscr{V}, \mathscr{F}) \\
& \cong \lim _{\mathscr{V} \in R(X)} C^{*}(\bar{M} \cap \mathscr{V}, \tilde{\mathscr{F}}) \\
& \cong \lim _{\mathscr{V} \in R(\bar{M})} C^{*}(\mathscr{V}, \tilde{\mathscr{F}})
\end{aligned}
$$

by (1) since $\bar{M}$ is closed in $X$; i.e. $\widetilde{C}^{*}(M, \mathscr{F}) \cong \check{C}^{*}(\bar{M}, \tilde{\mathscr{F}})$ and thus

$$
\widetilde{H}^{*}(M, \mathscr{F}) \cong \check{H}^{*}(\bar{M}, \tilde{\mathscr{F}}) \text {. }
$$

This shows in particular that the restricted cohomology may differ from the true cohomology. For if $X$ is a 2-sphere and $M=X-p$ where $p$ is any point 
of $X$, and if $Z$ is the simple sheaf of integers over $M$, then $\tilde{Z}$ is the simple sheaf of integers over $\bar{M}=X$; therefore $\widetilde{H}^{2}(M, Z) \cong \breve{H}^{2}(X, Z) \neq 0$, while

$$
H^{2}(M, Z)=0 \text {. }
$$

The relation between the restricted and true cohomologies of a subspace can in general be expressed in terms of a spectral sequence, according to the following theorem.

Theorem 2. If $\mathscr{F}$ is a sheaf over $M \subset X$ then there exists a spectral sequence $\left\{E_{r}\right\}$ satisfying isomorphisms

and

$$
E_{2}^{n, 0} \cong \tilde{H}^{n}(M, \mathscr{F}) \quad n \geqq 0
$$

$$
E_{\infty}^{p, q} \cong \mathscr{G}^{p} H^{p+q}(M, \mathscr{F}) \quad p, q \geqq 0
$$

for some filtration of $H^{*}(M, \mathscr{F})$.

Proof. Consider the double complex $K^{*} *=\tilde{C}^{*}\left(M, \mathscr{L}^{*}\right)$, where $\mathscr{L}^{*}$ $=\mathscr{C}^{*}(M, \mathscr{F})$ is the canonical flabby resolution (3) II 4.3.

Since the operations of taking sections, direct products and direct limits are left exact at least, the exact sequence of sheaves $0 \rightarrow \mathscr{F} \stackrel{j_{1}}{\rightarrow} \mathscr{L}^{0} \rightarrow \mathscr{L}^{1}$ gives an exact sequence of groups $0 \rightarrow \widetilde{C}^{p}(M, \mathscr{F}) \stackrel{j_{1}}{\rightarrow} \widetilde{C}^{p}\left(M, \mathscr{L}^{0}\right) \rightarrow \widetilde{C}^{p}\left(M, \mathscr{L}^{1}\right)$ each $p \geqq 0$, which shows that

$$
j_{1}: \tilde{C}^{*}(M, \mathscr{F}) \rightarrow \widetilde{C}^{*}\left(M, \mathscr{L}^{*}\right)
$$

embeds $\tilde{C}^{*}(M, \mathscr{F})$ as the subcomplex of $d_{2} 0$-cocycles of $K^{*}, *$; and therefore

$$
H^{0} K^{p, *} \cong \tilde{C}^{p}(M, \mathscr{F}) \text {. }
$$

Similarly the exact sequence (3) II 5.2.1. $0 \rightarrow \mathscr{L}^{q} \stackrel{j_{2}^{2}}{\rightarrow} \mathscr{C}^{0}\left(M \cap \mathscr{V}, \mathscr{L}^{q}\right) \rightarrow$ $\mathscr{C}^{1}\left(M \cap \mathscr{V}, \mathscr{L}^{q}\right)$ for each $\mathscr{V} \in R(X)$ and $q \geqq 0$, gives an embedding

$$
j_{2}: C^{*}(M, \mathscr{F}) \rightarrow \tilde{C}^{*}\left(M, \mathscr{L}^{*}\right)
$$

of $C^{*}(M, \mathscr{F})$ as the subcomplex of $d_{1} 0$-cocycles of $K^{*}, *$.

But

$$
\begin{aligned}
H^{q} K^{*, p} & =H^{q} \tilde{C}^{*}\left(M, \mathscr{L}^{p}\right) \\
& =H^{q} \lim _{\mathscr{V} \in R(X)} C^{*}\left(M \cap \mathscr{V}, \mathscr{L}^{p}\right) \\
& \cong \lim _{\mathscr{V} \in R(X)} H^{q}\left(M \cap \mathscr{V}, \mathscr{L}^{p}\right) \\
& =0 \quad(\text { all } q>0)
\end{aligned}
$$

by (3) II 5.2.3. since $\mathscr{L}^{p}$ is flabby all $p \geqq 0$; and therefore applying (3) we see that (7) induces an isomorphism all $n \geqq 0$

$$
j_{2}: H^{n}(M, \mathscr{F}) \cong H^{n}\left(K^{*}{ }^{*}\right) .
$$

If $\left\{E_{r}\right\}$ is the spectral sequence of $K^{*}, *$ with respect to its first filtration, then $E_{2}^{n, 0} \cong{ }^{\prime} H^{n \prime \prime} H^{0} K^{*^{\prime}, *^{\prime \prime}} \cong \tilde{H}^{n}(M, \mathscr{F})$ by $(6) ;$ and $E_{\infty}^{p, q} \cong \mathscr{G}^{p} H^{p+q}(K) \cong \mathscr{G}^{p} H^{p+q}(M, \mathscr{F})$ 
where $H^{*}(M, \mathscr{F})$ is filtered by the isomorphism (8) and the first filtration of $K^{*, *}$. $\left\{E_{r}\right\}$ is therefore a spectral sequence satisfying the required conditions.

Corollary. There exists a homomorphism $\tilde{H}^{*}(M, \mathscr{F}) \rightarrow H^{*}(M, \mathscr{F})$. This is bijective if $\bar{M}$ is paracompact and if $\lim _{U \in \psi(x)} H^{n}(M \cap U, \mathscr{F})=0$ for all $n>0$, each $x \in \bar{M} ;$ where $\Psi(x)$ is the directed set of open neighbourhoods of $x$ in $\bar{M}$, ordered by inclusion.

Proof. The spectral sequence of the previous theorem defines a homomorphism, (3) I 4.5. $E_{2}^{n,} \rightarrow H^{n}(K)$ i.e. $\widetilde{H}^{n}(M, \mathscr{F}) \rightarrow H^{n}(M, \mathscr{F})$ each $n \geqq 0$. This is bijective if the sequence degenerates, i.e. if $E_{2}^{p, q}=0$ all $q>0$.

Now

$$
\begin{aligned}
H^{q} \tilde{C}^{p}\left(M, \mathscr{L}^{*}\right) & \cong H^{q} \check{C}^{p}\left(\bar{M}, \tilde{\mathscr{L}}^{*}\right) \text { by (4) } \\
& =H^{q} \lim _{\mathscr{V} \in R(\bar{M})} \prod_{s \in S_{p}(\mathscr{V})} \tilde{\mathscr{L}}^{*}(|s|) \\
& \cong \lim _{\mathscr{V}} \prod_{s} H^{q} \mathscr{L}^{*}(M \cap|s|) \\
& \cong \lim _{\mathscr{V}} \prod_{s} H^{q}(M \cap|s|, \mathscr{F})
\end{aligned}
$$

by (3) II Lemma 4.9.1. since each $M \cap|s|$ is open in $M$ and $\mathscr{L}^{*}$ is the canonical resolution of $\mathscr{F}$ over $M$.

Thus $H^{q} \widetilde{C}^{p}\left(M, \mathscr{L}^{*}\right) \cong \check{C}^{p}\left(\bar{M}, \mathscr{H}^{q}\right)$ where $\mathscr{H}^{q}$ denotes the presheaf over $\bar{M}$ : $\mathscr{H}^{q}(U)=H^{q}(M \cap U, \mathscr{F})$; and therefore

$$
\begin{aligned}
E_{2}^{p, q} & \cong{ }^{\prime} H^{\prime \prime} H^{q} \tilde{C}^{*^{\prime}}\left(M, \mathscr{L}^{* \prime}\right) \\
& \cong \breve{H}^{p}\left(\bar{M}, \mathscr{H}^{q}\right) \\
& =0 \quad(\text { all } q>0)
\end{aligned}
$$

by (3) II 5.10.2. since the sheaf generated (3) II 1.2. by $\mathscr{H}^{q}$ over $\bar{M}$ has stalk over $x: \mathscr{H}^{q}(x)=\lim _{U \in \psi(x)} H^{q}(M \cap U, \mathscr{F})$ which is given to be zero all $x \in \bar{M}, q>0$.

\section{Relation to the Leray Sequence}

In (3) theorems II 5.4.1. and II 5.2.4. the Leray spectral sequence of an open, or a closed locally finite, covering $\mathscr{U}$ is given satisfying isomorphisms $E_{2}^{p, q} \cong$ $H^{p}\left\{\mathscr{U}, \mathscr{H}^{q}(\mathscr{F})\right\}$ and $E_{2}^{p, q} \cong \mathscr{G}^{p} H^{p+q}(X, \mathscr{F})$ where $\mathscr{H}^{q}(\mathscr{F})$ denotes the system of coefficients $s \rightarrow H^{q}(|s|, \mathscr{F})$.

If $\mathscr{U}$ is closed and $X$ paracompact the Cech and restricted and true cohomology groups of $|s|$ are all isomorphic for simplexes $s$ of $\mathscr{U}$. The sequence of Theorem 1 will then be isomorphic to the Leray sequence. In the case of an open covering we have the following result.

Theorem 3. If $\mathscr{U}$ is a locally finite open covering there exists a map of spectral sequences from the sequence of Theorem 1 to the Leray sequence of (3) II 5.4.1. This is induced in the $E_{2}$ terms by the map of local coefficients over $\mathscr{U}: \tilde{\mathscr{H}}^{*}(\mathscr{F})$ $\rightarrow \mathscr{H}^{*}(\mathscr{F})$ defined by the homomorphism of the corollary to Theorem 2. 
Proof. Consider the double complexes $K_{1}^{* * *}=C^{*}\left(\mathscr{U}, \tilde{\mathscr{C}}^{*}(\mathscr{F})\right\}$ and $K_{2}^{*, *}$ $=C^{*}\left\{\mathscr{U}, \mathscr{C}^{*}(X, \mathscr{F})\right\} ;$ and the triple complex $K^{*, *, *}=C^{*}\left\{\mathscr{U}, \tilde{\mathscr{C}}^{*}\left[\mathscr{C}^{*}(X, \mathscr{F})\right]\right\}$, the latter having differentiations $d_{1}, d_{2}$ and $d_{3}$. The sequences of Theorem 1 and of (3) II 5.4.1. are the spectral sequences of like $K_{1}$ and $K_{2}$ respectively with respect to their first filtrations.

The embeddings (5) and (7):

$$
\tilde{C}^{*}(|s|, \mathscr{F}) \stackrel{j_{1}}{\rightarrow} \widetilde{C}^{*}\left[|s|, \mathscr{C}^{*}(X, \mathscr{F})\right] \stackrel{j_{2}}{\leftarrow} C^{*}(|s|, \mathscr{F})
$$

for each $s \in S_{p}(\mathscr{U})$, give embeddings:

i.e.

$$
C^{*}\left\{\mathscr{U}, \tilde{\mathscr{C}}^{*}(\mathscr{F})\right\} \stackrel{j_{1}}{\rightarrow} C^{*}\left\{\mathscr{U}, \tilde{\mathscr{C}}^{*}\left[\mathscr{C}^{*}(X, \mathscr{F})\right]\right\} \stackrel{j_{2}}{\longleftarrow} C^{*}\left\{\mathscr{U}, \mathscr{C}^{*}(X, \mathscr{F})\right\}
$$

We have used the fact (3) II Lemma 4.9.1. that $\left.\mathscr{C}^{*}(X, \mathscr{F})\right|_{|s|} \cong \mathscr{C}^{*}(|s|, \mathscr{F})$ since $\mathscr{U}$ is open.

In the induced map of total cohomologies of (9):

$$
\widetilde{H}^{q}(|s|, \mathscr{F}) \stackrel{j_{1}}{\rightarrow} H^{q}\left\{\tilde{C}^{*}\left[|s|, \mathscr{C}^{*}(X, \mathscr{F})\right]\right\} \stackrel{j_{2}}{\leftarrow} H^{q}(|s|, \mathscr{F}),
$$

we have from (8) that $j_{2}$ is bijective, and by (3)

$$
j_{2}^{-1} \cdot j_{1}: \tilde{H}^{q}(|s|, \mathscr{F}) \rightarrow H^{q}(|s|, \mathscr{F})
$$

is the homomorphism of the corollary to Theorem 2.

Let $\mathscr{K}^{*, *}$ be the double complex defined by $\mathscr{K}^{p, q}=\sum_{q^{\prime}+r^{\prime}=q} K^{p, q^{\prime}, r^{\prime}}$ with differentiations $d_{1}$ and $d_{2}+d_{3}$. Then (10) defines maps of double complexes

$$
K_{1}^{*, *} \stackrel{j_{1}}{\rightarrow} K^{*, *} \stackrel{j_{2}}{\stackrel{2}{2}} K_{2}^{*, *}
$$

and hence, by (1) XV 6., maps of spectral sequences

$$
' E\left(K_{1}\right) \stackrel{j_{1}}{\rightarrow} E(\mathscr{K}) \stackrel{j_{2}}{\leftarrow} E\left(K_{2}\right),
$$

taking the first filtration of each double complex.

The $E_{2}^{p, q}$ terms in (13) are

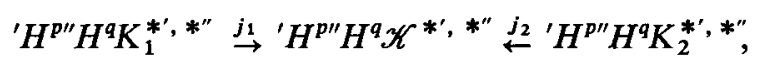

which are just the maps of the $p$ th cohomology of $\mathscr{U}$ induced by the maps of local coefficients (11) over simplexes $s$ of $\mathscr{U}$. Therefore $j_{2}$ in (14) is bijective and hence, by (1) XV 3.2., $j_{2}$ in (13) is an isomorphism of spectral sequences.

Thus $j_{2}^{-1}, j_{1}:{ }^{\prime} E\left(K_{1}\right) \rightarrow{ }^{\prime} E\left(K_{2}\right)$ is a map of spectral sequences induced in the $E_{2}$ terms by the maps of local coefficients (12) for simplexes $s$ of $\mathscr{U}$; which completes the proof of the theorem.

In conclusion, I wish to thank Sir William Hodge for much helpful advice. 


\section{REFERENCES}

(1) H. Cartan and S. Eilenberg, Homological Algebra (Princeton, 1956).

(2) S. EILENberg and N. Steenrod, Foundations of Algebraic Topology (Princeton, 1952).

(3) R. Godement, Théorie des Faisceaux (Paris, 1958).

(4) J. P. Serre, Faisceaux Algebriques Cohérents, Ann. of Math., 61, (1955).

DePartmeNT OF MATHEMATICS

UNIVERSITY OF GLASGOW 